\title{
An experience of developing Quran ontology with contextual information support.
}

\begin{abstract}
Purpose - Ontologies play an important role in enabling machines to understand and navigate a knowledge base. Currently, a number of ontologies covering specific topic in the Quran have been created to serve a particular application. However, these existing Quran ontologies are limited in scope and knowledge. Specifically, existing ontologies do not support contextual information that is considered necessary for correct interpretation of the verses of Quran. The paper aims to discuss these issues. Design/methodology/approach - In order to fill this gap, this paper reports development of an ontology for Juz' Amma that encapsulates contextual information support, which are the translations, revelations place, tafsir, and hadiths. Findings - The developed ontology was evaluated and was found to satisfy the requirements specification. Originality/value - In addition, this ontology can be reused and further enhanced to support many Quran-related semantic applications in the future.
\end{abstract}

Keyword: Ontology engineering; Quran corpus; Quran ontology. 